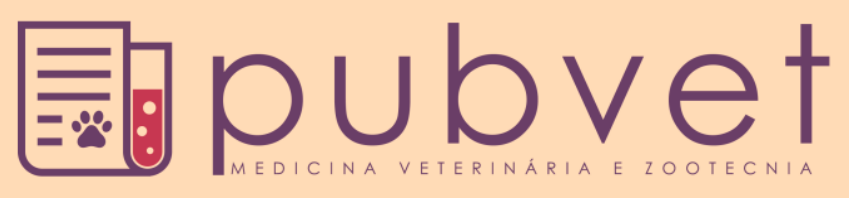

HTTP://DX.DOI.ORG/10.22256/PUBVET.V11N1.78-81

\title{
Piometra e hiperplasia vaginal em cadela: Relato de caso
}

\author{
Karina de Kássia da Silva Sales ${ }^{1}$, Nhirneyla Marques Rodrigues ${ }^{2}$, Andressa Kelly Barbosa \\ Rufino $^{3}$, Pedro Márcio da Silva $\mathrm{Luz}^{4}$ \\ ${ }^{I}$ Médica Veterinária, formada pela Universidade Federal do Piauí; Especialista em Clínica e Cirurgia de Cães e Gatos- Qualittas. \\ carinaksales@gmail.com \\ ${ }^{2}$ Mestre em Ciência Animal - UFPI; Residência Multiprofissional em Medicina Veterinária (Clinica Medica e Cirurgia de cães e gatos) - \\ UFPI.nhirneyla@hotmail.com \\ ${ }^{3}$ Acadêmica de Medicina Veterinária, Universidade Federal do Piauí. andressa_kbr@hotmail.com \\ ${ }^{4}$ Acadêmico de Medicina Veterinária, Universidade Federal do Piauí. pedromarcio_silva@hotmail.com
}

RESUMO. Na rotina médico-veterinária de cães e gatos há uma grande ocorrência de enfermidades que acometem o trato reprodutivo desses animais, acometendo em grande parte as fêmeas, podendo trazer consequências sérias como a infertilidade. Dentre as muitas patologias reprodutivas, citam-se com grande casuística a piometra (hiperplasia endometrial cística) e menos comumente a hiperplasia vaginal. A piomeira tende a ocorrer mais frequentemente em cadelas de meia idade ou animais idosos. Já a hiperplasia vaginal, acomete cadelas jovens devido à ação de estrogênio. Objetivou se com o presente estudo, relatar o caso de uma cadela, envolvendo ambas patologias. $\mathrm{O}$ tratamento de escolha realizado foi uma ovariosalpingohistectomia $(\mathrm{OSH})$ e a paciente recuperou-se bem. Conclui-se que emergências reprodutivas requerem um diagnóstico adequado e tratamento mais precoce possível para evitar maiores danos à saúde no animal. A OSH mostrou-se efetiva e curativa nestas patologias.

Palavras chave: Ciclo-estral, estrogênio, ovariosalpingohisterectomia, infecção

\section{Pyometra and vaginal hyperplasia in bitch: Case report}

ABSTRACT. In the medical-veterinary routine of dogs and cats there is a high occurrence of diseases that affect the reproductive tract of the animals, affecting mostly females, can have serious consequences such as infertility. Among the many reproductive pathologies, they cite yourself with large casuistry the pyometra (cystic endometrial hyperplasia) and less commonly the vaginal hyperplasia. The piomeira tends to occur most often in middleaged or elderly dogs animals. Already vaginal hyperplasia, affects young dogs due to estrogen action. The objective with this study, report the case of a bitch, involving both diseases. The treatment of choice made was one ovariosalpingohistectomia $(\mathrm{OSH})$ and the patient recovered well. It was concluded that reproductive emergencies require proper diagnosis and early treatment possible to avoid further damage to health in the animal. The OSH proved to be effective and curative in these pathologies.

Keywords: Cyclo-estrus, estrogen, ovariosalpingohisterectomy, infection

\section{Piómetra e hiperplasia vaginal en perra: Reporte de un caso}

RESUMEN. En la rutina del médico veterinario en perros y gatos frecuentemente se presentan enfermedades que afectan el sistema reproductivo de éstos animales, acometiendo en gran parte a las hembras, pudiendo traer consecuencias serias como la infertilidad. Entre las patologías reproductivas, se pueden con gran casuística a piómetra (hiperplasia endometrial cística) y menos comúnmente la hiperplasia vaginal. El piómetra tiende a ocurrir frecuentemente en perras de edad media o animales de edad avanzada. Ya 
la hiperplasia vaginal, acomete perras jóvenes debido a la acción de estrógeno. El objeto del presente estudio fue relatar el caso de una perra, envolviendo Piómetra e hiperplasia vaginal. El tratamiento de preferencia realizado fue una ovariosalpingohistectomia (OSH) e la paciente se recuperó muy bien. En conclusión, emergencias reproductivas requieren un diagnóstico adecuado y tratamiento inmediato para evitar mayores daños a la salud en el animal. A OSH mostró ser efectiva y curativa en este cao.

Palabras clave: Ciclo estral, estrógeno, ovariosalpingohisterectomia, infección

\section{Introdução}

$\mathrm{Na}$ rotina veterinária a incidência de enfermidades que acometem o trato reprodutivo de cães e gatos é grande. Acomete em grande parte as fêmeas, o que pode trazer consequências graves à saúde e ainda podem torná-las inférteis. Enumeram-se diversas doenças reprodutivas em fêmeas, tais como: distocias, piometra (hiperplasia endometrial cística), hiperplasia vaginal e anormalidades de origem congênita. Destas, a piometra e a hiperplasia vaginal são corriqueiramente diagnosticadas (Fontbonne, 2011). A piometra ocorre principalmente em cadelas de meia idade a idosas, trazendo sérios riscos à vida. Isso se dá em virtude dos repetidos ciclos estrais (Pretzer, 2008). Na maioria delas, a infecção tem participação de organismos de origem urinária ou fecal, principalmente Escherichia coli, podendo estar envolvidos também Staphylococcus sp., Citrobacter koseri, Enterobacter cloacae, Enterobacter faecalis, Eduardsiella sp e Klebsiella pneumoniae (Kalenski et al., 2012). Os níveis séricos de progesterona durante o diestro, fase onde comumente acontece a piometra, estimulam a proliferação endometrial e criam um ambiente propício para adesão e acúmulo bacteriano no útero, agravando o quadro clínico da paciente (Conrado, 2009).

$\mathrm{Na}$ hiperplasia vaginal, tem-se uma maior ocorrência em cadelas jovens. Esta afecção decorre da ação do estrogênio e macroscopicamente mostra-se como uma projeção da parede vaginal para o exterior, através da rima vulvar (Slatter, 2007). Algumas complicações ocorrem no tecido vaginal quando este é projetado durante o prolapso, como ulceração da mucosa vaginal, além de automutilação.

Mediante o exposto, objetivou-se com o presente estudo, relatar um caso de uma cadela portadora de hiperplasia vaginal e piometra concomitantemente.

\section{Relato de caso}

Foi atendida no Hospital Veterinário da Universidade Federal do Piauí (HVU-UFPI) uma cadela, sem raça definida (SRD), com três anos, não castrada. Ao exame clínico observou-se que o animal apresentava inapetência, apatia e hiperplasia vaginal (Figura 1), há dois dias, estando o tecido bastante hiperêmico e fragilizado.

O proprietário relatou que esta condição ocorreu outras vezes, mas com menor gravidade e melhora espontânea. Havia também, presença de secreção mucopurulenta de odor fétido. De imediato, procedeu-se com a limpeza da região genital do animal com clorexidine e solução fisiológica, a fim de evitar possíveis danos à mucosa vaginal, como infecções e necrose.

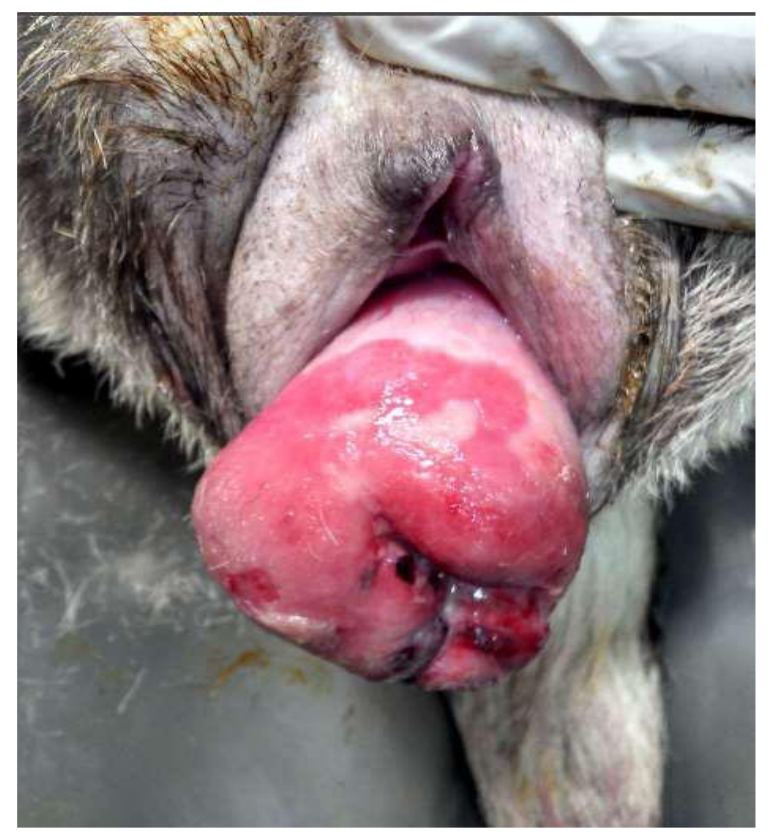

Figura 1. Aspecto macroscópico da hiperplasia vaginal da cadela, atendida no HVU.

Realizaram-se como exames complementares a citologia vaginal e ultrassonografia abdominal, cujo, laudo ultrassonográfico revelou que o animal apresentava infecção uterina, suspeitando-se de piometra, também denominada como síndrome hiperplásica endometrial cística (Figura 2). No 
mesmo dia, realizou-se a coleta de sangue para a realização de exames hematológicos (hemograma e bioquímico sérico) sendo estes necessários para a avaliação do estado fisiológico do animal, utilizados como exames pré-operatórios. Os resultados dos exames hematológicos estão descritos abaixo:

Hemograma - Anemia normocítica normocrômica. Leucocitose com neutrofilia, monocitose e eosinopenia absolutas. Trombocitoepenia.

Bioquímico sérico (Ureia, creatinina, fosfatase alcalina, ALT, proteínas totais, albumina e globulina) - sem alterações.

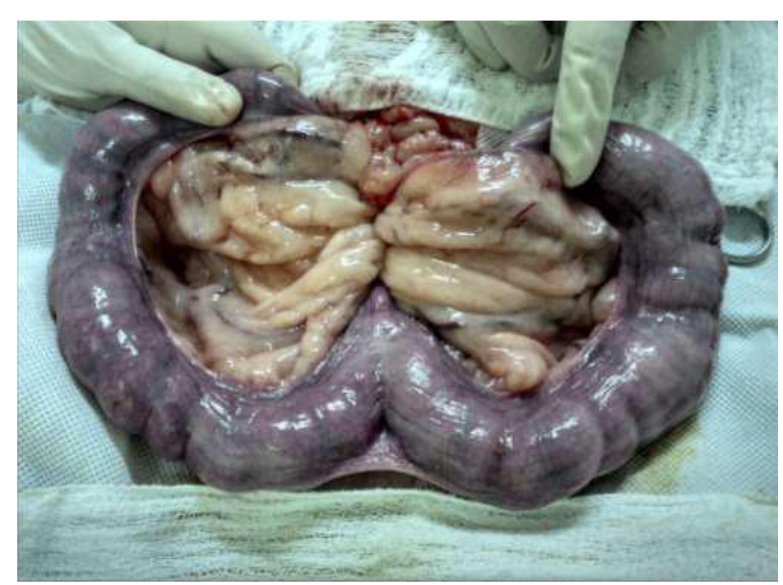

Figura 2. Aspecto macroscópico do útero com a síndrome hiperplásica endometrial cística (Piometra) da cadela, atendida no HVU.

A conduta terapêutica adotada foi a ovariosalpingohisterectomia (OSH). Como medicação pré-anestésica foi administrada: cefalotina $\quad(25 \mathrm{mg} / \mathrm{kg} / \mathrm{EV}), \quad$ tramadol (4 $\mathrm{mg} / \mathrm{kg} / \mathrm{IM})$. Na indução foram administrados midazolam $(0,3 \mathrm{mg} / \mathrm{kg} / \mathrm{IM})$, propofol (3 $\mathrm{mg} / \mathrm{kg} / \mathrm{EV})$ e manutenção anestésica com isoflurano em circuito semi-aberto.

A paciente foi posicionada em decúbito dorsal e já em plano anestésico procedeu-se a antissepsia do sítio cirúrgico. A técnica cirúrgica utilizada foi a tradicional, descrita na literatura (Fossum, 2014). A incisão foi pré retroumbilical na linha média. Os ovários foram localizados e exteriorizados. Os pedículos ovarianos foram ligados com nylon 3-0. A artéria uterina foi ligada bilateralmente. $\mathrm{O}$ útero foi ligado por transfixação (sutura de Parker-kerr) e os órgãos foram retirados. Todas as ligaduras foram feitas com fio de nylon 2-0. A cavidade foi fechada em três planos. O primeiro abrangeu peritônio, fáscia e músculos, utilizando-se sutura com pontos em
' $\mathrm{X}$ ' ou em pontos únicos separados. O segundo abrangeu o tecido subcutâneo e foi realizado com pontos tipo cushing. A pele foi fechada com pontos separados simples com categute. Todas as suturas foram feitas com fio de nylon 2-0. A intervenção cirúrgica foi realizada com sucesso.

No pós-cirúrgico o animal recebeu fármacos para controle da dor, inflamação e possíveis infecções. A analgesia foi realizada com (tramadol $3 \mathrm{mg} / \mathrm{kg}$, por via oral, sendo administrado a cada 8 horas; meloxicam $0,1 \mathrm{mg} / \mathrm{kg}$, via oral, a cada 24 horas) e antibioticoterapia (cefalexina $30 \mathrm{mg} / \mathrm{kg}$, via oral, a cada 12 horas; rifocina tópica na ferida) pós cirúrgica. $\mathrm{O}$ animal recuperou-se bem.

\section{Discussão}

O animal tinha três anos de idade e não recebia drogas contraceptivas. No entanto, a paciente foi portadora de duas condições reprodutivas emergenciais, fato incomum e que necessitou de um tratamento breve. Justifica-se nesse caso, a ocorrência de piometra, em virtude a ascensão de bactérias quando havia a abertura cervical durante proestro e estro, além dessa exposição vaginal repetida. Já que não fazia uso de contraceptivos injetáveis, que são citados como uma causa bem como para esta enfermidade (Souza et al., 2014).

A bactéria comumente observada em casos de piometra é a gram negativa Escherichia coli, pois esta tem maior afinidade pelo endométrio e miométrio. Essa alteração acomete normalmente fêmeas de meia idade a idosas, principalmente a partir dos seis anos, porém animais jovens podem desenvolver a doença, caso estes receberam injeções contraceptivas de estrógeno (Gonçalves, 2012).

No exame citológico, obeservou-se que a cadela já se encontrava em fase de diestro, fase citada como a de ocorrência da piometra (Birchard \& Sherding, 2008). Manteve-se o tecido vaginal exposto limpo e lubrificado de modo a minimizar traumas, uma vez que se não forem adotadas tais condutas, o mesmo pode ate se tornar necrótico (Freshman, 2013).

Conforme citado na literatura, optou-se pela OSH como forma curativa das doenças (Silveira et al., 2013), evitando recidivas e maiores complicações. Nos casos de piometra, o animal deve ser estabilizado antes da intervenção cirúrgica. Ou seja, é necessário que seja feita uma fluidoterapia intravenosa adequada e manutenção do equilíbrio hidroeletrolítico, devendo proceder também com uma adequada antibioticoterapia de 
amplo espectro para controle ou prevenção de sepse.

A conduta adotada para a hiperplasia vaginal de limpeza da região genital do animal com clorexidine e solução fisiológica foi realizada corretamente. Nesses casos o tecido edematoso exposto deve ser protegido contra traumas e infecção, se a mucosa for acometida, pode ser utilizado antibióticos tópicos e ainda colar elisabetano. Essa condição é autolimitante, pois o problema é solucionado quando não há mais influencia estrogênica no final do estro ou após a OSH (Nelson \& Couto, 2015).

No hemograma realizado, observou-se que a cadela apresentou anemia normocítica normocrômica, leucocitose com neutrofilia, monocitose e eosinopenia absolutas e trombocitopenia, colaborando com relatos na literatura (Evangelista, 2009); porém não é raro encontrar hemogramas sem alterações. $\mathrm{O}$ exame bioquímico sérico (Uréia, creatinina, fosfatase alcalina, ALT, proteínas totais, albumina e globulina), não apresentou alterações, indicando que o animal não havia comprometimento renal.

\section{Conclusão}

As afecções reprodutivas são comuns na rotina veterinária, podendo o animal apresentar apenas uma afecção ou duas afecções concomitantemente, como foi o caso da cadela atendida no Hospital Veterinário, que apresentou hiperplasia vaginal e piometra. Para que haja a recuperação eficiente do paciente é necessário que se tenha um diagnóstico adequado e o tratamento mais precoce possível, evitando-se maiores danos à saúde do animal. E diante da problemática apresentada pelo animal, a conduta em se realizar a ovariosalpingohisterectomia, se mostrou efetiva e curativa nestas patologias abordadas.

\section{Referências Bibliográficas}

Birchard, S. J. \& Sherding, R. G. 2008. Manual Saunders: clínica de pequenos animais, São Paulo.

Conrado, F. O. 2009. Aspectos clínicopatológicos da piometra. Faculdade de Medicina Veterinária. Universidade Federal do Rio Grande do Sul, Porto Alegrene.

Evangelista, L. S. M. 2009. Alterações clínicas e laboratoriais em cadelas com piometra antes e após ovariossalpingohisterectomia. Veterinária. Universidade Federal de Teresina, Teresina.
Fontbonne, A. 2011. Infertility in bitches and queen: recent advances. Revista Brasileira de Reproducao Animal, 35, 202-209.

Fossum, T. W. 2014. Cirurgia de pequenos animais, 4 edn. Elsevier Brasil, São Paulo.

Freshman, J. L. 2013. Hiperplasia e prolapso vaginal. In: Mazzaferro, E. M. (ed.) Emergências e cuidados críticos em pequenos animais. Roca, São Paulo.

Gonçalves, R. P. M. 2012. Coagulograma em cadelas com piometria e síndrome da resposta inflamatória sistêmica (SRIS). Veterinária. Universidade Federal do Paraná, Curitiba.

Kalenski, T. A., Reinoldes, A., Kitsis, M., Faustino, M., Talib, M. S. F. \& Cortopassi, S. R. G. 2012. Identificação das bactérias envolvidas na sepse grave de fêmeas caninas com piometra submetidas a ováriohisterectomia terapêutica. Brazilian Journal of Veterinary Research and Animal Science, 49, 130-138.

Nelson, R. W. \& Couto, C. G. 2015. Medicina interna de pequenos animais. Elsivier Editora, Amsterdan.

Pretzer, S. D. 2008. Clinical presentation of canine pyometra and mucometra: a review. Theriogenology, 70, 359-363.

Silveira, C. P., Machado, E. A. A., Silva, W. M., Marinho, T. C. M. S., Ferreira, A. R. A., Bürger, C. P. \& Costa Neto, J. M. 2013. Estudo retrospectivo de ovariossalpingo-histerectomia em cadelas e gatas atendidas em Hospital Veterinário Escola no período de um ano.

Slatter, D. H. 2007. Manual de cirurgia de pequenos animais. Manole, São Paulo.

Souza, J. P. M., Moraes, L. A., Pereira, J. M. M., Silva, S. P., Casseb, L. M. N. \& Casseb, A. d. R. 2014. Uso de contraceptivos de origem hormonal e quadro hematológico na incidência da piometra canina. Veterinária e Zootecnia, 21, 275-278.

\section{Article History:}

Received 10 October 2016

Accepted 17 November 2016

Available on line 9 December 2016

License information: This is an open-access article distributed under the terms of the Creative Commons Attribution License 4.0, which permits unrestricted use, distribution, and reproduction in any medium, provided the original work is properly cited. 